\title{
Differential Space-Time Coding Scheme Using Star Quadrature Amplitude Modulation Method
}

\author{
Xiangbin $\mathrm{Yu}^{1}{ }^{1} \mathrm{DaZhuan} \mathrm{Xu}{ }^{1,2}$ and Guangguo $\mathrm{Bi}^{2}$ \\ ${ }^{1}$ Information and Communication Engineering Postdoctoral Research Station, Nanjing University of Aeronautics and Astronautics, \\ Nanjing 210016, China \\ ${ }^{2}$ National Mobile Communications Research Laboratory, Southeast University, Nanjing 210096, China
}

Received 5 July 2005; Revised 11 January 2006; Accepted 13 January 2006

Recommended for Publication by Richard Barton

Differential space-time coding (DSTC) has received much interest as it obviates the requirement of the channel state information at the receiver while maintaining the desired properties of space-time coding techniques. In this paper, by introducing star quadrature amplitude modulation (star QAM) method, two kinds of multiple amplitudes DSTC schemes are proposed. One is based on differential unitary space-time coding (DUSTC) scheme, and the other is based on differential orthogonal space-time coding (DOSTC) scheme. Corresponding bit-error-rate (BER) performance and coding-gain analysis are given, respectively. The proposed schemes can avoid the performance loss of conventional DSTC schemes based on phase-shift keying (PSK) modulation in high spectrum efficiency via multiple amplitudes modulation. Compared with conventional PSK-based DSTC schemes, the developed schemes have higher spectrum efficiency via carrying information not only on phases but also on amplitudes, and have higher coding gain. Moreover, the first scheme can implement low-complexity differential modulation and different code rates and be applied to any number of transmit antennas; while the second scheme has simple decoder and high code rate in the case of 3 and 4 antennas. The simulation results show that our schemes have lower BER when compared with conventional DUSTC and DOSTC schemes.

Copyright $\odot 2006$ Hindawi Publishing Corporation. All rights reserved.

\section{INTRODUCTION}

With the fast development of modern communication technique, the demand for reliable high data rate transmission in fading channel is increased significantly, which stimulate much interest in multiple antennas communication, especially, space-time coding schemes [1-3]. However, the effectiveness of most space-time coding schemes depends on perfect channel estimation at the receiver, which is difficult to implement in practice due to rapid changes in time-varying channel, or due to the overhead needed to estimate a large number of parameters such as in a MIMO system [4]. Thus, the differential modulation scheme becomes an attractive alternative.

With differential detection, channel state information (CSI) is not required either at the transmitter or at the receiver. Hochwald and Marzetta [5] proposed an effective modulation scheme to improve system capacity (i.e., unitary space-time modulation) with noncoherent detection for multiple antennas in fading channel, and then unitary spacetime coding. Subsequently, Hochwald and Sweldens [6] and
Hughes [7] independently came up with differential unitary space-time coding scheme based on group codes. This scheme can allow easy implementation at the transmitter due to the application of group codes, but has higher decoding complexity. Afterwards, Tarokh and Jafarkhani [8] developed a simple differential space-time coding scheme based on STBC, but the scheme is limited in two transmit antennas. Thus in a subsequent work, they proposed a multiple antennas space-time coding scheme with more than 2 transmit antennas [9], but the scheme was still limited in existing STBC structure. The code-rate is only $1 / 2$, and corresponding decoding method is also complex. After the above work, Ganesan and Stoica firstly developed a simple but effective orthogonal space-time coding (OSTC) scheme [10] and then corresponding differential OSTC scheme [11]. Compared with the above-mentioned differential schemes, this scheme, with lower computational complexity while higher code rate in the case of three or four antennas, does not need the algebra group structure. Unfortunately, the above schemes basically need to employ the multiple levels phase shift-keying (MPSK) constellation, namely, the transmitted symbols are 
all from the unitary constellation and corresponding encoded matrix has unity-amplitude. As a result, when spectrum efficiency gets higher, the minimum product distance between the two encoded matrices decreases as the minimum distance between symbols decrease, which will bring about the obvious reduce of coding gain and the loss of system performance. These conclusions can also be achieved from Table I in [7] and Table I in [11]. Considering that the minimum distance between multiple levels QAM symbols is larger than the corresponding PSK symbols' distance [12], we adopt the star QAM method to map MPSK-based code matrices. On one hand, we can utilize both the phase and amplitude to carry information to improve the spectrum efficiency further; on the other hand, by star QAM method, constellation matrices are no longer limited in unity constellation and they have more freedom, the minimum produced distance is increased accordingly. Thus we can improve the performance of pervious code matrix and avoid the performance degradation in high spectrum efficiency. Although a differential space-time block code scheme based on squareQAM is proposed in [13], the scheme is limited in existing STBC structure and suits square STBC only, and has highcomplexity differential modulation. Motivated by the reason above, on the basis of analyzing differential orthogonal space-time coding (DOSTC) scheme and differential unitary space-time code (DUSTC) scheme, we develop the two multiple-amplitude differential space-time coding schemes by the star QAM method, and analyze corresponding performance over Rayleigh fading channel. Meanwhile, we give differential space-time coding scheme for nonsquare code matrix and derive the calculation formulas of the coding gain in detail. Compared with existing DUSTC and DOSTC, the schemes have lower bit error rate (BER) and higher coding gain.

\section{SYSTEM MODEL AND STAR QAM}

In this section, we consider a wireless communication system with $K$ antennas at the transmitter, $N$ antennas at the receiver, and the system operating over a flat Rayleigh fading channel. Given that $H=\left\{h_{k n}\right\}$ is $K \times N$, fading channel matrix, where $h_{k n}$ denotes the complex channel gain from transmit antenna $k$ to receive antenna $n$. The channel gains are modeled as samples of independent complex Gaussian random variables with zero-mean and variance 0.5 per real dimension, and the channel state information is unknown at the receiver. Let $G_{i}$ be the code matrix with $K \times K$ transmitted at time block $i$, then at the receiver, the received signal matrix $X_{i}$ can be expressed by

$$
X_{i}=\sqrt{\gamma} G_{i} H+Z_{i}
$$

where $Z_{i}$ is $K \times N$ complex Gaussian noise matrix, whose elements are independent, identically distributed (i.i.d) complex Gaussian random variables with zero-mean and unitvariance. Let the code matrix index be $i$ and time epoch index within the code matrix be $t$. So at the receiver, the received
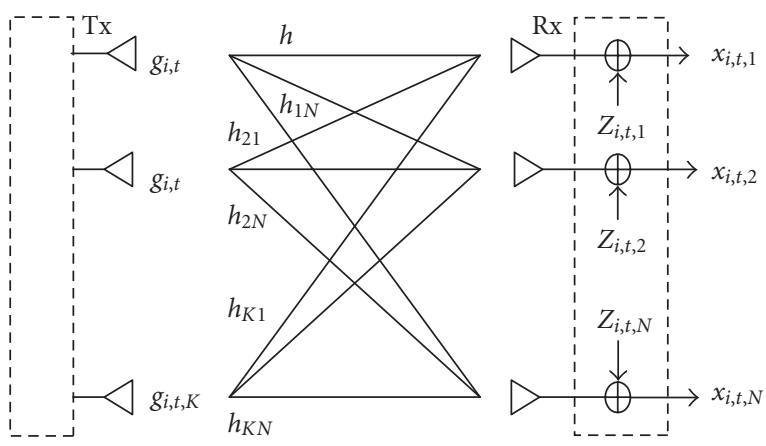

FIgURE 1: Structure diagram of a MIMO wireless system.

signal for receive antenna $n(n=1,2, \ldots, N)$ can be written by

$$
x_{i, t, n}=\sqrt{\gamma} \sum_{k=1}^{K} g_{i, t, k} h_{k, n}+z_{i, t, n},
$$

where the coded symbols from code matrix are normalized to obey $E\left\{\sum_{k=1}^{K}\left|g_{i, t, k}\right|^{2}\right\}=1$, thus it can ensure that $\gamma$ is the expected signal-to-noise ratio (SNR). $\left\{z_{i, t, n}\right\}$ are elements of noise matrix $Z_{i}$. The structure diagram of a MIMO wireless communication system with $K$ transmit antennas and $N$ receive antennas is illustrated in Figure 1.

As we know, quadrature amplitude modulation (QAM) is a bandwidth efficient transmission method for digital signals. Compared with MPSK modulation, the MQAM has stronger ability against inference in fading channel, its $M$ constellation points are not limited in unit amplitude, but have multiple amplitudes $[14,15]$. Thus they have more freedom and higher minimum distance among constellation points. For 4 bits symbol, there are usually two constellation mapping methods, that is, square mapping and star mapping. 16 square QAM requires coherent detection, whereas 16 star QAM can adopt differential detection. Due to the robustness of the differential detection scheme in fading channels, star QAM has received much attention for mobile radio applications [15]. For this reason, we employ the star QAM scheme to improve the performance of existing differential space-time codes. Figure 2 illustrates the signal constellations of $M$-level star QAM, it consists of two rings. The two rings both correspond to $M / 2$-PSK constellation, but they have different amplitudes, that is, $a_{0}$ and $a_{1}$ in Figure 2; where $a_{1}=\beta a_{0}, \beta$ is the amplitude ratio. For simplicity, $M=16$ is employed in Figure 2.

\section{MULTIPLE AMPLITUDES DIFFERENTIAL UNITARY SPACE-TIME CODING}

The differential encoding and decoding schemes using star QAM method for unitary space-time coding are analyzed in this section. The differential unitary space-time coding scheme can be applied to multiple antennas system with an arbitrary number of transmit antennas, and is very effective in the absence of channel state information at the receiver. At 


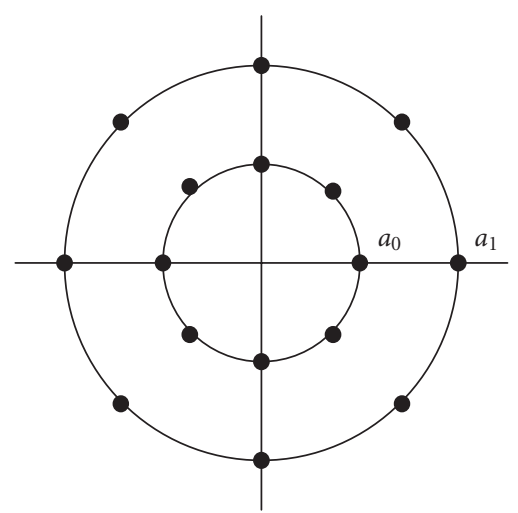

FIGURE 2: 16 star QAMs constellation.

the transmitter, the information is differentially encoded at time block $i$ as follows:

$$
G_{i}=V_{d_{i}} G_{i-1},
$$

where $G_{i}$ and $G_{i-1}$ are the transmitted matrices at $i$ and $i-1$, respectively; and initial code matrix $G_{0}=I_{K \times K}$, which does not carry any information. $V_{d_{i}}$ is a unitary information matrix. According to [6], for a transmission rate of $R$ bits/channel use, it requires a constellation with $P=2^{R K}$ different signals, each signal is a $K \times K$ unitary matrix $V_{p}$ from a constellation $\Upsilon$ of $P$ such distinct unitary matrices. Here, the data to be transmitted are assumed to be an integer data sequence $d_{1}, d_{2}, \ldots$, with $d_{p} \in\{0,1, \ldots, P-1\}$. Clearly, all the transmitted matrices $G_{i}$ will be unitary. While for [7], the information matrix $V_{i}$ is from the set of all possible information $\Gamma, \Gamma$ is $K \times K$ unitary matrix group. For any $V \in \Gamma$, the equation $V V^{H}=V^{H} V=I_{K \times K}$ holds; the superscript ${ }^{H}$ denotes conjugate transpose of matrix. Thus the transmitted signal matrix satisfies the equation $G_{i}=V_{i} G_{i-1}$ with initial matrix $G_{0}=D, D$ is a $K \times K$ unitary matrix [7].

At the receiver, we assume that the channel gains remain constant at two consecutive time blocks, then according to (1), the received matrices at time block $i-1$ and $i$ are give by

$$
X_{i-1}=\sqrt{\gamma} G_{i-1} H+Z_{i-1}, X_{i}=\sqrt{\gamma} G_{i} H+Z_{i}, \quad \text { respectively. }
$$

From (3), (4) can be changed as follows:

$$
\begin{aligned}
X_{i} & =\sqrt{\gamma} G_{i} H+Z_{i} \\
& =\sqrt{\gamma} V_{d_{i}} G_{i-1} H+Z_{i} \\
& =V_{d_{i}}\left(X_{i-1}-Z_{i-1}\right)+Z_{i} \\
& =V_{d_{i}} X_{i-1}+Z_{i}-V_{d_{i}} Z_{i-1} \\
& =V_{d_{i}} X_{i-1}+\sqrt{2} \tilde{Z}_{i},
\end{aligned}
$$

where $\tilde{Z}_{i}$ is a $K \times N$ noise matrix. Consider that $Z_{i}$ and $Z_{i-1}$ are both complex Gaussian matrices and their elements are zero-mean and unit-variance, and $\tilde{Z}_{i}$ is a complex Gaussian matrix, and its elements are also i.i.d complex Gaussian random variables with zero-mean and unit-variance.

From the above-mentioned analysis and [6], we can obtain the decision variable for transmitted data $d_{i}$ by employing maximum likelihood (ML) detector as follows:

$$
\begin{aligned}
\hat{d}_{i} & =\arg \min _{p=0, \ldots, P-1}\left\|X_{i}-V_{p} X_{i-1}\right\|^{2} \\
& =\arg \min _{p=0, \ldots, P-1} \operatorname{tr}\left\{\left(X_{i}-V_{P} X_{i-1}\right)\left(X_{i}-V_{P} X_{i-1}\right)^{H}\right\},
\end{aligned}
$$

where the operator $\operatorname{tr}(\cdot)$ denotes the matrix trace.

Considering

$$
\begin{aligned}
\operatorname{tr}\left\{\left(X_{i}\right.\right. & \left.\left.-V_{p} X_{i-1}\right)\left(X_{i}-V_{p} X_{i-1}\right)^{H}\right\} \\
= & \operatorname{tr}\left\{X_{i} X_{i}^{H}\right\}-2 \operatorname{Re}\left\{\operatorname{tr}\left(V_{p} X_{i-1} X_{i}^{H}\right)\right\} \\
& +\operatorname{tr}\left\{V_{p} X_{i-1} X_{i-1}^{H} V_{p}^{H}\right\} \\
= & \operatorname{tr}\left\{X_{i} X_{i}^{H}\right\}-2 \operatorname{Re}\left\{\operatorname{tr}\left(X_{i-1} X_{i}^{H} V_{p}\right)\right\} \\
& +\operatorname{tr}\left\{X_{i-1} X_{i-1}^{H} V_{p}^{H} V_{p}\right\} .
\end{aligned}
$$

Then (6) can be equivalent to

$$
\hat{d}_{i}=\arg \max _{p \in 0, \ldots, P-1} \operatorname{Re}\left\{\operatorname{tr}\left(X_{i-1} X_{i}^{H} V_{p}\right)\right\} .
$$

Based on the above analysis, the ML detector for differential modulation can be interpreted as follows: the block code received at time $i-1$ is used as an estimate of the channel, and this estimate is used to do a coherent detection of the block code at time $i$. Similarly, the block code received at time $i$ is also the channel estimate for decoding block $i+1$.

To simplify the transmission scheme and the constellation designs, [6] also gives simple group code structure. Namely, the set $\left\{V_{0}, \ldots, V_{P-1}\right\}$ forms a cyclic group, and $V_{p}=V_{1}^{P}\left(p=0,1, \ldots, P ; V_{1}\right.$ is a diagonal generator matrix $)$ becomes a diagonal matrix, this design criterion is essentially the same as the scheme in [7]. Thus according to [7], the decision value for the information matrix $V_{i}$ can be achieved by employing ML detector as follows:

$$
\begin{aligned}
\hat{V}_{i} & =\arg \min _{V_{i} \in \Phi}\left\|X_{i}-V_{i} X_{i-1}\right\|^{2} \\
& =\arg \min _{V_{i} \in \Phi} \operatorname{tr}\left\{\left(X_{i}-V_{i} X_{i-1}\right)\left(X_{i}-V_{i} X_{i-1}\right)^{H}\right\} \\
& =\arg \max _{V_{i} \in \Phi} \operatorname{Re}\left\{\operatorname{tr}\left(X_{i-1} X_{i}^{H} V_{i}\right)\right\},
\end{aligned}
$$

where constellation $\Phi$ is the set formed by $\left\{V_{0}, \ldots, V_{P-1}\right\}$; as shown in $[6,7], \Phi$ has group structure, and the optimal codes are achieved by maximizing the coding gain in $\Phi$. The coding gain (as defined in [11]) is

$$
\Lambda_{c g}=\min _{F u \neq F q} K \times \Lambda_{p}\left(F_{u}, F_{q}\right),
$$


where $\Lambda_{p}\left(F_{u}-F_{q}\right)=\left\{\operatorname{det}\left(\left(F_{u}-F_{q}\right)\left(F_{u}, F_{q}\right)^{H}\right)\right\}^{1 / K}$ is the product distance between two code matrices $F_{u}$ and $F_{q}$, $\operatorname{det}(\cdot)$ representing determinant operator.

For a constellation constructed from multiple data symbols, a good metric to judge the performance is the square of the minimum distance between two points in the constellation. If the distance is bigger, then the performance is better. Similarly, for the constellation constructed by code matrices, the coding gain (i.e., above $\Lambda_{c g}$ ) is a good metric to judge the performance of corresponding constellation in terms of error probability analysis in $[6,7]$. For unitary space-time coding, however, when spectrum efficiency gets higher, the performance will become worse due to the lower coding gain, which can be seen in Table I in [7]. Considering that the matrices in unitary space-time codes group has unity energy (i.e., single amplitude), which can be thought as MPSK constellation, whereas star MQAM scheme has better performance than corresponding MPSK under the same spectrum efficiency, we adopt the star QAM scheme to design superior DUSTC. By using the star QAM method, we can carry information by means of not only the phase but also the amplitude of the code matrix. The spectral efficiency is thus improved accordingly. Moreover, the constellation matrices will not be limited in unity-energy, thus they have different amplitudes, and minimum product distance will be improved accordingly. As a result, the performance degradation is overcome effectively in high spectrum efficiency. Specific encoding and decoding schemes are designed as follows.

At the transmitter, the input bit streams are divided into each data block including $\log _{2} M$ bits, namely the $i$ th data block corresponds to data bits $\left\{b_{i m}, m=1,2, \ldots, \log _{2} M\right\}$. The first bit $b_{i 1}$ is used to decide the amplitude of differential unitary space-time code matrix to transmit, other $\log _{2} M$ 1 bits $\left\{b_{i m}, m=2, \ldots, \log _{2} M\right\}$ perform conventional differential unitary space-time modulation, and the modulation will adopt group code which takes values in the $M / 2$-PSK rather than MPSK. Then, we design corresponding multipleamplitude differential encoding scheme in terms of the following equations:

$$
G_{i}^{\prime}=\left|G_{i}^{\prime}\right| G_{i}=\left|G_{i}^{\prime}\right| V_{i} G_{i-1}, \quad G_{i}=V_{i} G_{i-1},
$$

where $\left|G_{0}^{\prime}\right|=\rho_{0}, G_{0}=I_{K \times K}[6]$ or $G_{0}=D$ [7], $\left|G_{i}^{\prime}\right|$ denotes the amplitude of $G_{i}^{\prime}$. It may choose $\rho_{0}$ or $\rho_{1}\left(\rho_{0}\right.$ and $\rho_{1}$ are the amplitudes of inner and outer unitary matrix constellation, which are similar to the amplitudes of inner and outer ring of star QAM scheme, respectively; and $\rho_{1}=\beta \rho_{0}$ ), which depends on the value of $b_{i 1}$. If $b_{i 1}=0$, the amplitude of $G_{i}^{\prime}$ is the same as that of the previous transmitted code matrix $G_{i-1}^{\prime}$; if $b_{i 1}=1$, the amplitude of $G_{i}^{\prime}$ is different from the amplitude of $G_{i-1}^{\prime}$, that is, if $\left|G_{i-1}^{\prime}\right|=\rho_{0},\left|G_{i}^{\prime}\right|=\rho_{1}$; and if $\left|G_{i-1}^{\prime}\right|=\rho_{1}$, $\left|G_{i}^{\prime}\right|=\rho_{0}$.

At the receiver, we employ the method similar to star QAM demodulation to demodulate the received signals. After multiple amplitudes modulation, the received signal matrices at time blocks $i-1$ and $i$ are changed accordingly as follows:

$$
\begin{gathered}
X_{i-1}=\sqrt{\gamma} G_{i-1}^{\prime} H+Z_{i-1}=\sqrt{\gamma}\left|G_{i-1}^{\prime}\right| G_{i-1} H+Z_{i-1}, \\
X_{i}=\sqrt{\gamma} G_{i}^{\prime} H+Z_{i}=\sqrt{\gamma}\left|G_{i}^{\prime}\right| V_{i} G_{i-1} H+Z_{i} .
\end{gathered}
$$

Based on the above received signal matrices, we can make corresponding differential detection to achieve the decision variables of $b_{i m}$ (i.e., $\hat{b}_{i m} m=1, \ldots, \log _{2} M$ ). Firstly, the phase detection is performed in terms of (9), namely conventional DUSTC demodulation method can be applied. Thus, corresponding decision bit variables $\hat{b}_{i m}\left(m=2, \ldots, \log _{2} M\right)$ are obtained. Then, we employ the detection method in [14] to perform amplitude detection. Namely, the decision variable $\hat{b}_{i 1}$ for amplitude bit is obtained according to the equivalent amplitude ratio

$$
\begin{aligned}
\lambda_{a} & =\sqrt{\frac{\sum_{t=1}^{K} \sum_{n=1}^{N}\left|x_{i, t, n}\right|^{2}}{\sum_{t=1}^{K} \sum_{n=1}^{N}\left|x_{i-1, t, n}\right|^{2}}} \\
& =\frac{\left\|X_{i}\right\|}{\left\|X_{i-1}\right\|},
\end{aligned}
$$

where $\left\|X_{i}\right\|$ is the Frobenius norm of $X_{i}$.

Let $\xi_{L}$ and $\xi_{H}$ be two amplitude decision thresholds as shown in [14], these decision thresholds are assumed to be related according to $\xi_{H}=1 / \xi_{L}$, and they satisfy the following conditions:

$$
\beta^{-1}<\xi_{L}<1, \quad 1<\xi_{H}<\beta .
$$

If $\lambda_{a}$ falls inside two decision thresholds, that is, if $\lambda_{a}$ satisfies $\xi_{L}<\lambda_{a}<\xi_{H}$, the amplitude decision variable $\hat{b}_{i 1}=0$. The converse holds, if $\lambda_{a}$ falls outside of two decision thresholds, that is, if $\lambda_{a}$ satisfies $\lambda_{a}<\xi_{L}$ or $\lambda_{a}<\xi_{H}, \hat{b}_{i 1}=1$. The above threshold values can be optimized so that corresponding system BER is minimized under a given SNR.

Based on the above analysis and property of unitary space-time codes, the proposed multiple amplitudes DUSTC scheme (MDUSTC) can implement full diversity and different rates; and it can be applied to any number of antennas. Besides, the scheme has low-complexity differential modulation due to the diagonal matrix of USTC, but it requires group structure and has exponential decoding complexity. Fortunately, [16] gives fast ML decoding algorithms for conventional USTC scheme. The algorithms exploit the constellation structures and are polynomial rather than exponential, in the rates $R$ and $K$.

\section{MULTIPLE AMPLITUDES DIFFERENTIAL ORTHOGONAL SPACE-TIME CODING}

\subsection{Code matrix}

In this section, taking the disadvantage of the above-mentioned MDUSTC scheme into account, we will give another multiple amplitudes differential orthogonal space-time coding (MDOSTC) scheme, which has simpler decoder and 
higher coding gain while it does not need a group structure in general.

Let $\left\{U_{l}\right\}_{l=1}^{L}$ and $\left\{W_{l}\right\}_{l=1}^{L}$ be a set of $2 L$ matrices of size $K \times K$ which satisfy the following conditions:

$$
\begin{gathered}
U_{l} U_{l}^{H}=I_{K \times K}, \quad W_{l} W_{l}^{H}=I_{K \times K}, \quad \forall l, \\
U_{l} U_{s}^{H}=-U_{s} U_{l}^{H}, \quad W_{l} W_{s}^{H}=-W_{s} W_{l}^{H}, \quad \forall l \neq s, \\
U_{l} W_{s}^{H}=W_{s} U_{l}^{H}, \quad \forall l, s,
\end{gathered}
$$

where $I_{K \times K}$ denotes identity matrix. Then $\left\{U_{l}\right\}$ and $\left\{W_{l}\right\}$ are said to constitute an amicable orthogonal design of order $K$ in $L$ variables $[10,11]$. The detailed design process for $\left\{U_{l}\right\}$ and $\left\{W_{l}\right\}$ with $K=2,4,8$ which meet with the conditions in (16) can be seen in [10]. By introducing the amicable orthogonal design, we can design corresponding code matrix.

Let $\left\{c_{i l}\right\}_{l=1}^{L}$ be a block of $L$ symbols to be transmitted at a time $i$, and the symbol $c_{i l}(l=1, \ldots, L)$ is from PSK constellation $\Omega$. Thus the $c_{i l}$ can be expressed by $c_{i l}=c_{i l}^{R}+j c_{i l}^{I}$, where $c_{i l}^{R}$ and $c_{i l}^{I}$ denote the real and imaginary parts of $c_{i l}$, respectively. By defining the $C_{i}$ as

$$
C_{i}=\frac{\sum_{l=1}^{L}\left(U_{l} c_{i l}^{R}+j W_{l} c_{i l}^{I}\right)}{\sqrt{L}}
$$

Then we have

$$
C_{i} C_{i}^{H}=\left(\frac{\sum_{l=1}^{L}\left|c_{i l}\right|^{2}}{L}\right) I_{K \times K}=I_{K \times K} .
$$

Thus $C_{i}$ is a unitary code matrix.

\subsection{Differential encoding and decoding schemes}

In this subsection, the differential encoding and decoding schemes for orthogonal space-time coding are firstly analyzed. Then multiple amplitudes DOSTC scheme using star QAM method is given. At the transmitter, we consider the case of $K \times K$ square code matrices at first. According to [10], we have such $K \times K$ matrices $\left\{U_{l}, W_{l}\right\}$ for $K=2,4$, and 8 . The $i$ th block to be transmitted is a differential encoding matrix $G_{i}$ with $K \times K$. At the start of the transmission, the transmitter sends a $K \times K$ identity matrix as initial code matrix $G_{0}$ (i.e., $G_{0}=I_{K \times K}$ ), which does not carry information. Then the information matrix $C_{i}$ as defined by (17) is differentially encoded in terms of $G_{i}=C_{i} G_{i-1}$. From $G_{0}=I_{K \times K}, C_{i}$ is unitary matrix, and $G_{i}=C_{i} G_{i-1}$, we can testify that $G_{i}$ is a unitary matrix. Thus the information matrix $C_{i}$ can be decoded from $G_{i} G_{i-1}^{H}=C_{i} G_{i-1} G_{i-1}^{H}=C_{i}$ if the code matrices $G_{i}$ and $G_{i-1}$ are observable at the receiver.

At the receiver, the received matrices at times $i$ and $i-1$ are written by $X_{i}=\sqrt{\gamma} G_{i} H+Z_{i}$ and $X_{i-1}=\sqrt{\gamma} G_{i-1} H+Z_{i-1}$, respectively. by

According to (9), we can obtain the ML detector for $\left\{c_{i l}\right\}$

$$
\left\{\hat{c}_{i l}\right\}_{l=1}^{L}=\arg \max _{\left\{c_{i l}\right\}, c_{i l} \in \Omega} \operatorname{Re}\left\{\operatorname{tr}\left(X_{i-1} X_{i}^{H} C_{i}\right)\right\}
$$

From (17), (19) can be further transformed to ML detector for single symbol $c_{i l}$ by

$$
\begin{aligned}
\widehat{c}_{i l}= & \arg \max _{c_{i l} \in \Omega} \operatorname{Re}\left\{\operatorname{tr}\left(X_{i-1} X_{i}^{H} U_{l}\right)\right\} c_{i l}^{R} \\
& +\operatorname{Re}\left\{\operatorname{tr}\left(j X_{i-1} X_{i}^{H} W_{l}\right)\right\} c_{i l}^{l} \\
= & \arg \max _{c_{i l} \in \Omega} \operatorname{Re}\left\{\operatorname{tr}\left(X_{i-1} X_{i}^{H} U_{l}\right)\right\} c_{i l}^{R} \\
& +\operatorname{Im}\left\{\operatorname{tr}\left(-X_{i-1} X_{i}^{H} W_{l}\right)\right\} c_{i l}^{I},
\end{aligned}
$$

where $\operatorname{Re}(\cdot)$ and $\operatorname{Im}(\cdot)$ denote real part operator and imaginary part operator, respectively. Equation (20) can be changed into the detection of real part and imaginary part in parallel as follows:

$$
\begin{gathered}
\hat{c}_{i l}^{R}=\arg \max _{c_{i l} \in \Omega} \operatorname{Re}\left\{\operatorname{tr}\left(X_{i-1} X_{i}^{H} U_{l}\right)\right\} c_{i l}^{R}, \\
\hat{c}_{i l}^{I}=\arg \max _{c_{i l} \in \Omega} \operatorname{Im}\left\{-\operatorname{tr}\left(X_{i-1} X_{i}^{H} W_{l}\right)\right\} c_{i l}^{I} .
\end{gathered}
$$

Namely the detector has a decoupled form, one scalar detector for each of the symbols $\left\{c_{i l}\right\}$. Thus compared with the detection method of other differential codes, the detection method of the proposed scheme has a much lower computational complexity.

From the above-mentioned analysis, we can see that differential orthogonal space-time coding scheme is still limited in MPSK modulation, and its performance will degrade under high spectrum efficiency. It is because corresponding de crease of minimum product distance between code matrices brings about the reduction of coding gain, and these conclusions can be drawn form Table I in [11]. Motivated by the reason analyzed in Section 3, we adopt the star QAM constellation method to map the code matrices to improve the performance of conventional DOSTC scheme. Specific encoding and decoding schemes are designed as follows.

At the transmitter, the input bits stream are divided into each data block including $\left(1+L \log _{2} M\right)$ bits, namely, $b_{i m}$ $\left(m=1,2, \ldots, 1+L \log _{2} M\right)$ represents the $i$ th data block, where $L$ is the number of symbols to be transmitted in the information code matrix. The first bit $b_{i 1}$ is used to decide the amplitude of differential orthogonal space-time code matrix; other bits firstly perform MPSK modulation, and are mapped to corresponding data symbols. Then these symbols are used to construct the information code matrix $C_{i}$ in terms of (17). Afterwards, the following differential modulation is performed:

$G_{i}^{\prime}=\left|G_{i}^{\prime}\right| G_{i}=\left|G_{i}^{\prime}\right| C_{i} G_{i-1}, \quad G_{i}=C_{i} G_{i-1}, \quad G_{0}=I_{K \times K}$. 
After performing multiple amplitudes modulation, the received code matrix need corresponding changes as shown in (13). Namely,

$$
X_{i}=\sqrt{\gamma}\left|G_{i}^{\prime}\right| G_{i} H+Z_{i}=\sqrt{\gamma}\left|G_{i}^{\prime}\right| C_{i} G_{i-1} H+Z_{i} .
$$

At the receiver, we make phase and amplitude detection for the received code matrices, respectively. At first, we employ conventional DOSTC decoding method to perform phase detection, namely, by utilizing (20) and (21) to get ML detector of $\left\{c_{i l}, l=1,2, \ldots, L\right\}$. Then via demapping in accordance, the decision bits $\left\{\hat{b}_{i m}, m=2, \ldots, 1+L \log _{2} M\right\}$ are obtained. For amplitude detection, we can adopt the decision method in Section 3. Based on this method, the amplitude decision bit $\hat{b}_{i 1}$ is finally achieved.

\subsection{Nonsquare matrix differential space-time coding and code rate}

The scheme presented in Section 4.2 is valid for $K=2,4$, and 8 transmit antennas, and corresponding code matrix is square matrix. Now we consider the scheme in the case of $K=3,5,6$, and 7 transmit antennas, where the code matrix will not be square matrix. This is also an open problem which needs to be solved in future work in [13]. For simplicity of analysis, we only focus on the 3 transmit antennas case; similar analysis can be extended to other three cases. For 3 transmit antennas, we transmit the first three columns of the differential code matrix of 4 transmit antennas to perform corresponding data transmission, which can be realized by a transform matrix as follows:

$$
G_{i}^{(3)}=G_{i}^{(4)} T,
$$

where $G_{i}^{(3)}$ and $G_{i}^{(4)}$ denote the transmitted differential code matrices for the case of 3 transmit antennas and 4 transmit antennas, respectively. $G_{i}^{(4)}$ is $4 \times 4$ square matrix, and $G_{i}^{(3)}$ is $4 \times 3$ nonsquare matrix; $T=\left[\begin{array}{lll}1 & 0 & 0 \\ 0 & 1 & 0 \\ 0 & 0 & 1 \\ 0 & 0 & 0\end{array}\right]$ is a $4 \times 3$ transform matrix.

Based on the above transform and (22), we can implement the data transmission of 3 transmit antennas case by transmitting $G_{i}^{(4)} T$. According to (23), the corresponding received signal matrix at time $i$ is written by

$$
X_{i}=\sqrt{\gamma}\left|G_{i}^{\prime(3)}\right| G_{i}^{(4)} T H+Z_{i}=\sqrt{\gamma}\left|G_{i}^{(3)}\right| G_{i}^{(4)} \tilde{H}+Z_{i},
$$

where $\tilde{H}=T H$ is the equivalent channel gain matrix. After equivalent transform, the $T$ can be absorbed in the channel gain matrix. Considering that $T^{H} T=I_{3 \times 3}$, then $\tilde{H}^{H} \tilde{H}=$ $H^{H} T^{H} T H=H^{H} H$. Thus the same diversity performances are obtained. Moreover, after the above transform, we can make use of the previous 4-antenna analysis method to detect the received data for 3-antenna systems at the receiver, and the system performance is not affected.

In addition, our scheme is based on the amicable orthogonal design, so the scheme has the same code rate of the code proposed in [10]. In the case of 2 transmit antennas; the code rate of our scheme is unity-rate, which is the same as the scheme proposed in [8]. Moreover, in the case of 3 or 4 transmit antennas, our scheme has a code rate of $3 / 4$, which is higher than the scheme developed in [9]; and the same as the schemes proposed in $[11,13]$, but the structure of code matrix is simpler than that in [13]. Besides, our scheme has lower computational complexity. However, we also notice that the scheme is only applied to no more than 8 transmit antennas due to the limitation of amicable orthogonal design, and in the case of more than 5 transmit antennas, it has only $1 / 2$-code rate, which will affect high date rate transmission to some extent and bring about the decrease of data rate.

\section{BIT-ERROR-RATE PERFORMANCE}

From the theory analysis in Sections 3 and 4, we can see that the phase and amplitude detection processes are independent, thus we can evaluate the average bit error rate (BER) via calculating the BER's of phase detection and amplitude detection separately. Namely,

$$
\begin{aligned}
& P_{b}=\frac{\left[\left(\log _{2} M-1\right) P_{b \text {-phase }}+P_{b \text {-amplitude }}\right]}{\log _{2} M} \text { for MDUSTC, } \\
& P_{b}=\frac{\left[\left(L \log _{2} M\right) P_{b \text {-phase }}+P_{b \text {-amplitude }}\right]}{1+L \log _{2} M} \text { for MDOSTC, }
\end{aligned}
$$

where $P_{b \text {-phase }}$ and $P_{b \text {-amplitude }}$ are the BER's of phase detection and amplitude detection, respectively. These equations are a weighted sum of the BER of phase detection and amplitude detection. ten by

For amplitude detection, the bit error probability is writ-

$$
\begin{aligned}
& P_{b \text {-amplitude }} \\
& \quad=\frac{\left[P_{b, a m}(H L)+P_{b, a m}(H H)+P_{b, a m}(L H)+P_{b, a m}(L L)\right]}{4},
\end{aligned}
$$

where $P_{b, a m}(H L)$ denotes the amplitude detection error probability for amplitude bits from outer constellation to inner constellation; other three items (i.e., $P_{b, a m}(H H)$, $\left.P_{b, a m}(L H), P_{b, a m}(L L)\right)$ can be explained in similar manner. These four terms may be further changed as

$$
\begin{gathered}
P_{b, a m}(H L)=P_{\rho_{1}, \rho_{0}}\left(\lambda_{a}>\xi_{L}\right)-P_{\rho_{1}, \rho_{0}}\left(\lambda_{a}>\xi_{H}\right), \\
P_{b, a m}(L H)=P_{\rho_{0}, \rho_{1}}\left(\lambda_{a}>\xi_{L}\right)-P_{\rho_{0}, \rho_{1}}\left(\lambda_{a}>\xi_{H}\right), \\
P_{b, a m}(H H)=P_{\rho_{1}, \rho_{1}}\left(\lambda_{a}>\xi_{H}\right)+1-P_{\rho_{1}, \rho_{1}}\left(\lambda_{a}>\xi_{L}\right), \\
P_{b, a m}(L L)=P_{\rho_{0}, \rho_{0}}\left(\lambda_{a}>\xi_{H}\right)+1-P_{\rho_{0}, \rho_{0}}\left(\lambda_{a}>\xi_{L}\right),
\end{gathered}
$$


where $P_{\rho_{1}, \rho_{0}}\left(\lambda_{a}>\xi_{L}\right)$ denotes the amplitude detection error probability for amplitude bits from outer constellation to inner constellation when $\lambda_{a}>\xi_{L}$; similar explanation can be applied for other seven items.

For phase detection, the bit error probability is written by

$P_{b \text {-phase }}=\frac{\left[P_{b, p h}(H L)+P_{b, p h}(H H)+P_{b, p h}(L H)+P_{b, p h}(L L)\right]}{4}$,

where $P_{b, p h}(H L)$ denotes the phase detection error bit probability for phase signal (which consists of corresponding phase bits) from outer constellation to inner constellation; other three items can be explained in similar way.

Consider that detailed BER derivation is more complex, and needs a plenty of mathematic calculation and theoretical analysis. Namely, it needs more space for BER calculation. Here, we only give some calculation steps to briefly review the derivation. For simplicity, the following only provides these steps with the example of 2Tx and 1Rx MDOSTC scheme.

(1) Calculate the phase detection error bit probability $P_{b \text {-phase }}$ in terms of (30).

From (30), we need to compute $P_{b, p h}(H L), P_{b, p h}(H H)$, $P_{b, p h}(L H)$, and $P_{b, p h}(L L)$, respectively. Considering that these four cases occur with equal probability, we can choose an arbitrary case for first calculation. Without loss of generality, $P_{b, p h}(H L)$ is firstly considered. By a series of calculation and using some related results from [12], we can derive its approximate parameter expression on amplitude pair $\left(\rho_{1}, \rho_{0}\right)$ (which corresponds to phase signal from outer constellation to inner constellation). Then employing similar calculation method, and substituting this amplitude pair with $\left(\rho_{1}, \rho_{1}\right)$, $\left(\rho_{0}, \rho_{1}\right)$, and $\left(\rho_{0}, \rho_{0}\right)$ in corresponding places, respectively, we can evaluate corresponding phase detection error bit probabilities $P_{b, p h}(H H), P_{b, p h}(L H)$, and $P_{b, p h}(L L)$, respectively. According to (30), we can obtain the $P_{b \text {-phase }}$ based on the evaluated $P_{b, p h}(H L), P_{b, p h}(H H), P_{b, p h}(L H)$, and $P_{b, p h}(L L)$.

(2) Evaluate the amplitude detection error bit probability $P_{b \text {-amplitude }}$ in terms of (28) and (29).

From (28), we need to calculate $P_{b, a m}(H L), P_{b, a m}(H H)$, $P_{b, a m}(L H)$, and $P_{b, a m}(L L)$, respectively. Without loss of generality, we choose $P_{b, a m}(H L)$ as first calculation. According to (29), $P_{\rho_{1}, \rho_{0}}\left(\lambda_{a}>\xi_{L}\right)$ and $P_{\rho_{1}, \rho_{0}}\left(\lambda_{a}>\xi_{H}\right)$ need to be calculated. Here, we first evaluate the amplitude detection error probability for amplitude bits from outer constellation to inner constellation when $\lambda_{a}>\xi_{L}$, that is, $P_{\rho_{1}, \rho_{0}}\left(\lambda_{a}>\xi_{L}\right)$ is firstly evaluated. According to (14) and employing the appendices results of [12], we can obtain the value of $P_{\rho_{1}, \rho_{0}}\left(\lambda_{a}>\xi_{L}\right)$ via a series of calculation and derivation. Using similar calculation method and substituting $\xi_{L}$ with $\xi_{H}$, we can calculate the value of $P_{\rho_{1}, \rho_{0}}\left(\lambda_{a}>\xi_{H}\right)$. Then changing the amplitude pair $\left(\rho_{1}, \rho_{0}\right)$ into $\left(\rho_{1}, \rho_{1}\right),\left(\rho_{0}, \rho_{1}\right)$, and $\left(\rho_{0}, \rho_{0}\right)$ in corresponding places, respectively, and utilizing the above calculation method, we can evaluate $P_{\rho_{1}, \rho_{1}}\left(\lambda_{a}>\xi_{L}\right), P_{\rho_{0}, \rho_{1}}\left(\lambda_{a}>\xi_{L}\right)$, and $P_{\rho_{0}, \rho_{0}}\left(\lambda_{a}>\xi_{L}\right)$, respectively. Thus according to the evaluated three values, we can obtain $P_{\rho_{1}, \rho_{1}}\left(\lambda_{a}>\xi_{H}\right), P_{\rho_{0}, \rho_{1}}\left(\lambda_{a}>\xi_{H}\right)$ and $P_{\rho_{0}, \rho_{0}}\left(\lambda_{a}>\xi_{H}\right)$ via substituting $\xi_{L}$ with $\xi_{H}$ accordingly. Based on the above results and (29), the values for $P_{b, a m}(H L)$,
$P_{b, a m}(H H), P_{b, a m}(L H)$, and $P_{b, a m}(L L)$ can be calculated, respectively. As a result, we will obtain the amplitude detection error bit probability $P_{b \text {-amplitude }}$ according to (28).

(3) Compute the average bit error rate for MDOSTC scheme.

Based on the obtained $P_{b \text {-phase }}$ and $P_{b \text {-amplitude, we can }}$ compute the average bit error rate for MDOSTC in terms of (27). Similar method can be applied to evaluate the average bit error rate for MDUSTC in terms of (26). Due to high calculation complexity, we no longer provide the average BER expression here; the detailed BER derivation will appear in another paper of ours for space considerations.

\section{CODING-GAIN ANALYSIS}

As mentioned in Section 3, for the constellation constructed by code matrices, the coding gain (i.e., $\Lambda_{c g}$ in (10)) is a good metric to judge the performance of corresponding constellation. By maximizing the coding gain, the optimal group codes are obtained in $[6,7]$. Let $F_{u}$ and $F_{q}$ be the information matrices (as defined in (17)) constructed from the data symbols sets $\left\{f_{u 1}, \ldots, f_{u L}\right\}$ and $\left\{f_{q 1}, \ldots, f_{q L}\right\}$, respectively, where symbols $\left\{f_{u l}\right\}$ and $\left\{f_{q l}\right\}$ are both from constellation $\Omega$. Let $F=F_{u}-F_{q}$, according to (17), $F$ can be changed to

$$
F=\frac{\sum_{l=1}^{L}\left[U_{l}\left(f_{u l}^{R}-f_{q l}^{R}\right)+j W_{l}\left(f_{u l}^{l}-f_{q l}^{l}\right)\right]}{\sqrt{L}} .
$$

Then according to (18), we have

$$
\begin{aligned}
F F^{H} & =\frac{1}{L}\left(\sum_{l=1}^{L}\left|\left(f_{u l}^{R}-f_{q l}^{R}\right)\right|^{2}+\left|\left(f_{u l}^{l}-f_{q l}^{l}\right)\right|^{2}\right) I_{k \times K} \\
& =\frac{1}{L}\left(\sum_{l=1}^{L}\left|f_{u l}-f_{q l}\right|^{2}\right) I_{k \times K} .
\end{aligned}
$$

So the product distance between $F_{u}$ and $F_{q}$ can be written by

$$
\begin{aligned}
\Lambda_{p}\left(F_{u}, F_{q}\right) & =\left\{\operatorname{det}\left(\left(F_{u}, F_{q}\right)\left(F_{u}, F_{q}\right)^{H}\right)\right\}^{1 / K}=\left\{\operatorname{det}\left(F F^{H}\right)\right\}^{1 / K} \\
& =\left\{\operatorname{det}\left(\frac{1}{L}\left(\sum_{l=1}^{L}\left|f_{u l}-f_{q l}\right|^{2}\right) I_{K \times K}\right)\right\}^{1 / K} \\
& =\frac{1}{L}\left(\sum_{l=1}^{L}\left|f_{u l}-f_{q l}\right|^{2}\right) .
\end{aligned}
$$

For $F_{u} \neq F_{q},(33)$ is minimized when $\left\{f_{u l}\right\}$ and $\left\{f_{q l}\right\}$ differ in just one symbol, while the other corresponding symbols are same, namely, the minimal value corresponds to the minimal distance between constellation points from $\Omega$. Hence, we can evaluate the coding gain in terms of (10) and (33) as follows:

$$
\begin{aligned}
\Lambda_{c g} & =\min _{f_{u l}, f_{m l} \in \Omega}\left(\frac{K}{L}\right)\left|f_{u l}-f_{q l}\right|^{2} \\
& =\left(\frac{K}{L}\right) d_{\min }^{2}=\left(\frac{K}{L}\right)\left[2 \sin \left(\frac{\pi}{M}\right)\right]^{2},
\end{aligned}
$$


where $d_{\min }$ is the minimal distance between constellation points of $M$-level PSK constellation $\Omega$. The above equation is used to calculate the coding gain of MPSK-based conventional DOSTC scheme. In the following, we will give another formula to evaluate the coding gain of the proposed MDOSTC scheme.

In this paper, we assume that the amplitude bits are transmitted with equal probability, and $\rho_{1}=\beta \sqrt{2 /\left(1+\beta^{2}\right)}$ so that $\left(\rho_{1}^{2}+\rho_{0}^{2}\right) / 2=1$, where $\rho_{0}=\rho_{1} / \beta$, and $\beta$ is assumed to be 2 , which is the optimum amplitude ratio from $[14,15]$, for this, we will give detailed explanation in simulation. According to (10), the coding gain of MDOSTC scheme can be defined as follows:

$$
\begin{aligned}
\Lambda_{c g} & =\min _{F_{u}^{\prime} \neq F_{q}^{\prime}} K \times \Lambda_{p}\left(F_{u}^{\prime}, F_{q}^{\prime}\right) \\
& =\min _{F_{u}^{\prime} \neq F_{q}^{\prime}} K \times \Lambda_{p}\left(\left|F_{u}^{\prime}\right| F_{u},\left|F_{q}^{\prime}\right| F_{q}\right),
\end{aligned}
$$

where $\left|F_{u}^{\prime}\right|$ and $\left|F_{q}^{\prime}\right|$ are the amplitudes of matrices $F_{u}^{\prime}$ and $F_{q}^{\prime}$, respectively; which choose $\rho_{0}$ or $\rho_{1}$.

When matrices $F_{u}^{\prime}$ and $F_{q}^{\prime}$ are from the same constellation (i.e., inner constellation or outer constellation), $\left|F_{u}^{\prime}\right|=\left|F_{q}^{\prime}\right|$. Without loss of generality, let they be from inner constellation, then we have

$$
\begin{aligned}
\Lambda_{p}\left(F_{u}^{\prime}, F_{q}^{\prime}\right) & =\left\{\operatorname{det}\left(\left(\rho_{0} F_{u}-\rho_{0} F_{q}\right)\left(\rho_{0} F_{u}-\rho_{0} F_{u}\right)^{H}\right)\right\}^{1 / K} \\
& =\rho_{0}^{2}\left\{\operatorname{det}\left(\left(F_{u}-F_{q}\right)\left(F_{u}-F_{q}\right)^{H}\right)\right\}^{1 / K} \\
& =\rho_{0}^{2} \Lambda_{p}\left(F_{u}, F_{q}\right) .
\end{aligned}
$$

From (34) and (36), (35) can be changed to

$$
\Lambda_{c g 0}=\left(\frac{K}{L}\right)\left[2 \rho_{0} \sin \left(\frac{\pi}{\frac{M}{2}}\right)\right]^{2} .
$$

Note: for MDOSTC scheme using $M$-level star QAM method, the symbols in matrices $F_{u}$ and $F_{q}$ are from $M / 2$ level PSK constellation.

Similarly, we can evaluate the coding gain when $F_{u}^{\prime}$ and $F_{q}^{\prime}$ are from outer constellation:

$$
\Lambda_{c g l}=\left(\frac{K}{L}\right)\left[2 \rho_{1} \sin \left(\frac{\pi}{\frac{M}{2}}\right)\right]^{2} .
$$

When matrices $F_{u}^{\prime}$ and $F_{q}^{\prime}$ are from different constellation, namely, one is from inner constellation, the other is from outer constellation. Without loss of generality, let the former be from outer constellation, and the latter from inner constellation, then $F_{u}^{\prime}-F_{q}^{\prime}$ is changed as follows:

$$
\begin{aligned}
\left(F_{u}^{\prime}-F_{q}^{\prime}\right) & =\left(\rho_{1} F_{u}-\rho_{0} F_{q}\right) \\
& =\frac{1}{\sqrt{L}} \sum_{l=1}^{L}\left[U_{l}\left(\rho_{1} f_{u l}^{R}-\rho_{0} f_{q l}^{R}\right)+j W_{l}\left(\rho_{1} f_{u l}^{I}-\rho_{0} f_{q l}^{I}\right)\right] .
\end{aligned}
$$

So according to (39) and (18), we have the following equation:

$$
\begin{aligned}
\left(F_{u}^{\prime}-\right. & \left.F_{q}^{\prime}\right)\left(F_{u}^{\prime}-F_{q}^{\prime}\right)^{H} \\
& =\frac{1}{L} \sum_{l=1}^{L}\left[\left|\rho_{1} f_{u l}^{R}-\rho_{0} f_{q l}^{R}\right|^{2}+\left|\rho_{1} f_{u l}^{I}-\rho_{0} f_{q l}^{I}\right|^{2}\right] I_{K \times K} \\
& =\frac{1}{L} \sum_{l=1}^{L}\left[\left|\rho_{1} f_{u l}-\rho_{0} f_{q l}\right|^{2}\right] I_{K \times K} .
\end{aligned}
$$

Hence,

$$
\begin{aligned}
\Lambda_{p}\left(F_{u}^{\prime}, F_{q}^{\prime}\right) & =\left\{\operatorname{det}\left(\left(F_{u}^{\prime}-F_{q}^{\prime}\right)\left(F_{u}^{\prime}-F_{q}^{\prime}\right)^{H}\right)\right\}^{1 / K} \\
& =\frac{1}{L} \sum_{l=1}^{L}\left[\left|\rho_{1} f_{u l}-\rho_{0} f_{q l}\right|^{2}\right] .
\end{aligned}
$$

Considering that $\left\{f_{u l}\right\}$ and $\left\{f_{q l}\right\}$ are from PSK constellation, we can assume that $f_{u l}=e^{j \theta_{u l}}$ and $f_{q l}=e^{j \theta_{q l}}$. Then we have

$$
\begin{aligned}
\left|\rho_{1} f_{u l}-\rho_{0} f_{q l}\right|^{2} & =\left|\rho_{1} e^{j \theta_{u l}}-\rho_{0} e^{j \theta_{q l}}\right|^{2} \\
& =\left(\rho_{1} e^{j \theta_{u l}}-\rho_{0} e^{j \theta_{q l}}\right)\left(\rho_{1} e^{-j \theta_{u l}}-\rho_{0} e^{-j \theta_{q l}}\right) \\
& =\rho_{1}^{2}+\rho_{0}^{2}-2 \rho_{1} \rho_{0} \cos \left(\theta_{u l}-\theta_{q l}\right) \\
& \geq \rho_{1}^{2}+\rho_{0}^{2}-2 \rho_{1} \rho_{0}=\left(\rho_{1}-\rho_{0}\right)^{2},
\end{aligned}
$$

where $\cos \left(\theta_{u l}-\theta_{q l}\right) \leq 1$ is utilized. So the minimum value of $\left|\rho_{1} f_{u l}-\rho_{0} f_{q l}\right|^{2}$ is $\left(\rho_{1}-\rho_{2}\right)^{2}$.

Based on the above analysis, using (41) and (42), we can evaluate the coding gain as follows:

$$
\begin{aligned}
\Lambda_{c g 2} & =\min _{F_{u}^{\prime} \neq F_{q}^{\prime}} K \times \Lambda_{p}\left(F_{u}^{\prime}, F_{q}^{\prime}\right) \\
& =K \times \frac{1}{L} \times L\left[\left(\rho_{1}-\rho_{0}\right)^{2}\right]=K\left(\rho_{1}-\rho_{0}\right)^{2} .
\end{aligned}
$$

Similarly, we can calculate the coding gain when $F_{u}^{\prime}$ and $F_{q}^{\prime}$ are from inner constellation and outer constellation, respectively:

$$
\Lambda_{c g 3}=\min _{F_{u}^{\prime} \neq F_{q}^{\prime}} K \times \Lambda_{p}\left(F_{u}^{\prime}, F_{q}^{\prime}\right)=K\left(\rho_{0}-\rho_{1}\right)^{2} .
$$

Considering equally likely the transmission of the amplitude bits, namely, the high and the low amplitude bits occur uniformly with a probability of 0.5 [15], the above four cases for calculating coding gain will occur with equal probability. Thus we can obtain the average coding gain of the proposed MDOSTC scheme using $M$-level star QAM method by 


$$
\begin{aligned}
\bar{\Lambda}_{c g} & =\frac{\left[\Lambda_{c g 0}+\Lambda_{c g l}+\Lambda_{c g 2}+\Lambda_{c g 3}\right]}{4} \\
& =\frac{\left\{\left(\frac{K}{L}\right)\left[2 \rho_{0} \sin \left(\frac{\pi}{\frac{M}{2}}\right)\right]^{2}+\left(\frac{K}{L}\right)\left[2 \rho_{1} \sin \left(\frac{\pi}{\frac{M}{2}}\right)\right]^{2}+2 K\left(\rho_{1}-\rho_{0}\right)^{2}\right\}}{4} .
\end{aligned}
$$

TABLE 1: Comparison of coding gain of DOSTC scheme and MDOSTC scheme.

\begin{tabular}{cccc}
\hline Constellation $\Phi$ & $\begin{array}{l}\text { Coding gain } \\
(K=2, L=2)\end{array}$ & $\begin{array}{l}\text { Coding gain } \\
(K=3, L=3)\end{array}$ & $\begin{array}{l}\text { Coding gain } \\
(K=4, L=3)\end{array}$ \\
\hline 10 PSK & 0.38197 & 0.38197 & 0.5093 \\
16 PSK & 0.1522 & 0.1522 & 0.203 \\
22 PSK & 0.081 & 0.081 & 0.108 \\
32 PSK & 0.0384 & 0.0384 & 0.05124 \\
16 star QAM & 0.6928 & 0.8928 & 1.1905 \\
32 star QAM & 0.4771 & 0.6771 & 0.9028 \\
\hline
\end{tabular}

Based on (34) and (45), we compare the coding gain of the multiple amplitudes DOSTC scheme and conventional DOSTC scheme under different spectrum efficiency in Table 1. From Table 1, we can see that the coding gain of the proposed MDOSTC scheme is higher than that of DOSTC scheme under same spectrum efficiency, while the coding gain of DOSTC scheme is higher than the corresponding differential unitary space-time coding scheme [11]. Hence our scheme has superior performance. Moreover, with the increase of spectrum efficiency, that is, when $M$ becomes bigger, the coding gain of conventional DOSTC scheme will decrease quickly; whereas for our scheme, the coding gain decreases slowly. So our scheme can avoid the extra performance degradation of conventional DOSTC scheme effectively in high spectrum efficiency.

For the presented multiple amplitudes DUSTC (MDUSTC) scheme, we can employ the above-mentioned analysis method and related computation formula from [7] to evaluate the coding gain, detailed deriving process is no longer given due to the repeated work. Similarly, the same conclusion can be reached. Namely, our MDUSTC scheme has higher coding gain than the corresponding DUSTC scheme under the same spectrum efficiency. Thus the performance of our MDUSTC scheme is superior to the corresponding DUSTC scheme, which will also be testified by the following simulation.

\section{SIMULATION RESULTS}

In this section, to test the validity of the proposed scheme, we provide the simulation results in Rayleigh fading channel. The channel is assumed to be quasistatic flat fading. In simulation, the differential unitary space-time coding and

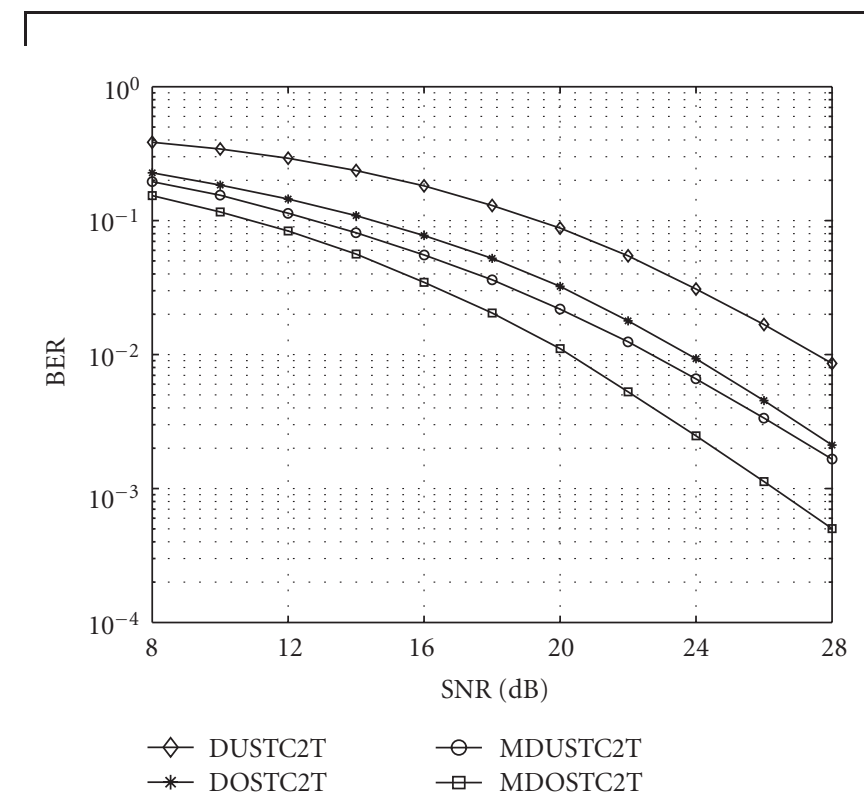

FIGURE 3: BER against SNR for different differential space-time codes with 2-transmit antennas.

differential orthogonal space-time coding are applied for the purpose of comparison with the same spectrum efficiency. Every data frame includes 960 information bits, and Gray mapping of the bits to symbol is employed. The numbers of transmit antennas are 2,3 , and 4 , and the number of receive antennas is set as 1 for simplicity. Besides, in the case of performing amplitude detection, we adopt the parameters given in $[14,15]$, such as $\beta=2, \xi_{H}=1.47, \xi_{L}=0.68$, and so forth. The simulation results are obtained from $10^{6}$ MonteCarlo simulation run, and they are illustrated in Figures 3-6, respectively.

Figure 3 shows the BER versus SNR for different differential space-time coding schemes with 2-transmit antennas. In Figure 3, the 16-level PSK modulation is applied to the DUSTC and the DOSTC, and the 16 star QAM method is applied to the multiple amplitudes DUSTC and the multiple amplitudes DOSTC. For the DUSTC, the generator matrix of the cyclic group codes $\operatorname{diag}(\exp (2 \pi j / 256), \exp (150 \pi j / 256))$ [6] is utilized, where "diag" and following "invdiag" denote the diagonal matrix and the inverse diagonal matrix, respectively; and $\rho_{1}\langle\operatorname{diag}(\exp (2 \pi j / 64), \exp (38 \pi j / 64))\rangle \cup$ $\rho_{2}\langle\operatorname{diag}(\exp (2 \pi j / 64), \exp (38 \pi j / 64))\rangle$ is used for MDUSTC. From Figure 3 , it shows that our MDOSTC scheme gives about $6 \mathrm{~dB}$ gains over the DUSTC and $2 \mathrm{~dB}$ over the DOSTC at a BER of $10^{-2}$. Moreover, another developed MDUSTC 


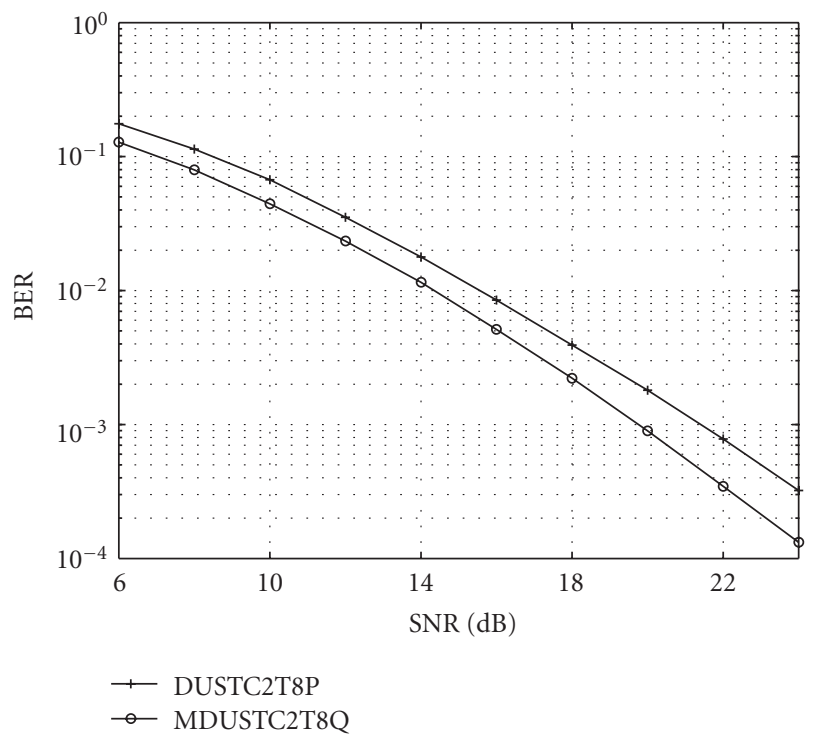

FIgURE 4: BER against SNR for DUSTC and MDUSTC with 2transmit antennas.

scheme also obtains about $3 \mathrm{~dB}$ gains over the DUSTC at $\mathrm{BER}=10^{-2}$. A similar conclusion can be drawn from Figure 4. In Figure 4, we compare the performance of the proposed MDUSTC scheme with the DUSTC scheme developed in [7], our scheme uses the 8 star QAM method, while the DUSTC employs the 8 PSK modulation; where the generator matrix of the optimal unitary group codes $\langle\operatorname{diag}(\exp (2 \pi j / 8), \exp (-2 \pi j / 8)), \operatorname{invdiag}(-1,1)\rangle[7]$ is used for the DUSTC, and the $\rho_{1}\langle\operatorname{diag}(\exp (2 \pi j / 4), \exp (-2 \pi j / 4))$, $\operatorname{invdiag}(-1,1) \cup \rho_{2}\langle\operatorname{diag}(\exp (2 \pi j / 4), \exp (-2 \pi j / 4))$, invdiag $(-1,1)\rangle$ is used for the MDUSTC. Similarly, our scheme still outperforms the corresponding DUSTC scheme; it achieves $2 \mathrm{~dB}$ gains at the BER of $10^{-3}$. So after adopting multiple amplitudes modulation, our developed schemes are both superior to corresponding differential space-time coding schemes, and our MDUSTC scheme slightly outperforms DOSTC. The reason for these is that our schemes can obtain higher coding gain via using star QAM method, which is consistent with the theoretical analysis in Section 6. Besides, the proposed MDOSTC scheme performs better than another proposed MDUSTC scheme, which accords with the conclusions drawn in [11], that is, DOSTC outperforms DUSTC.

In the above work, we employ the parameters in $[14,15]$. In fact, these decision threshold (i.e., $\xi_{H}, \xi_{L}$ ) and amplitude ratio $\beta$ can be chosen and optimized in terms of the criterion that the system BER is minimized at given SNR. Using this criterion, the optimum $\beta$ for differential detection of single antenna system in Rayleigh fading channel was found to be approximately 2; and the threshold values were searched and calculated, they were 1.47 and 0.68 , respectively [15]. Similarly, according to this criterion, [14] gave the optimized values about $\beta$ and $\xi_{H}\left(\xi_{L}=1 / \xi_{H}\right)$ under Rician fading channel by computer search, that is, $\beta=2, \xi_{H}=1.47$. In our work, we also optimize these decision thresholds for multiple amplitudes differential space-time code scheme via computer

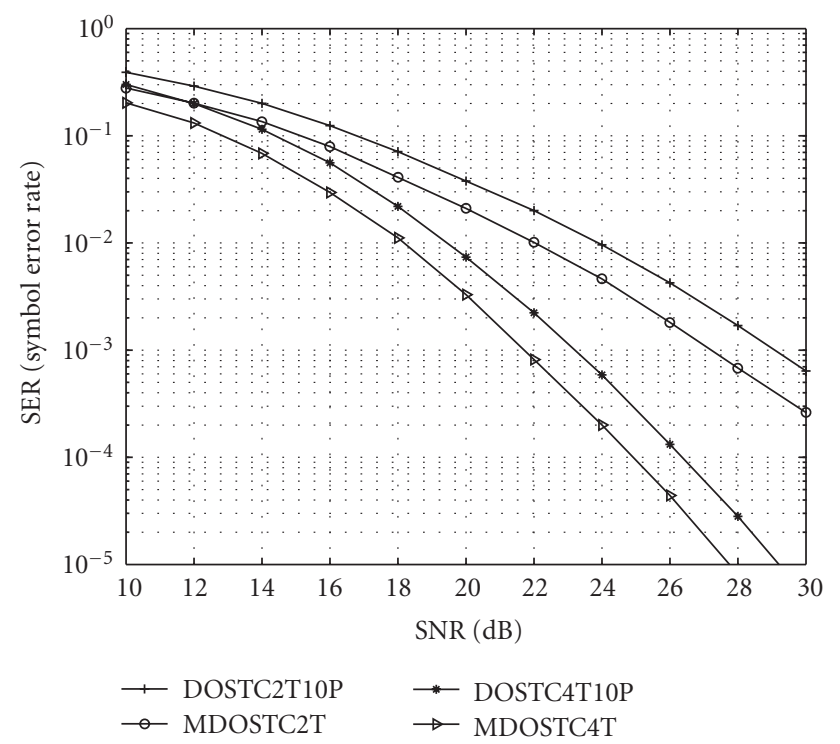

FIGURE 5: SER against SNR for DOSTC and MDOSTC with 2transmit antennas and 4-transmit antennas.

search. Firstly, the optimized amplitude ratio is produced in terms of the above criterion by fixing $\xi_{H}=1.47$, then using this optimized amplitude ratio, the threshold values are reevaluated and optimized so that the system BER is minimized, the detailed optimized process can be seen in $[14,15]$. Of course, we may also obtain the optimum combination of $\left(\beta, \xi_{H}\right)$ via computer search based on the above optimum criterion. As a result, the produced optimized value is almost identical to the optimized values provided by $[14,15]$, so we still adopt the above parameters in following simulation.

In addition, we also notice that for MDOSTC scheme, its real spectrum efficiency is $\left(1+L \log _{2} 8\right) / L=3.5$ with $L=2$, and $\left(1+L \log _{2} 8\right) / L=3.33$ with $L=3$. To maintain the same spectrum efficiency, the 10 PSK modulation is applied to DOSTC, which has spectrum efficiency of 3.32. Based on these, we give symbol error rate (SER) comparison between MDOSTC scheme and DOSTC with 2-transmit antennas and 4-transmit antennas under the same SNR. The results are shown in Figure 5, where "DOSTC2T10P" and "DOSTC4T10P" represent the differential orthogonal space-time coding based on 10 PSK with 2-transmit antennas and 4-transmit antennas, respectively. "MDOSTC2T" and "MDOSTC4T" represent the multiple amplitudes DOSTC using 16 star QAM method with 2-transmit antennas and 4transmit antennas, respectively. From Figure 5, we observe that proposed MDOSTC schemes still outperform corresponding differential orthogonal space-time coding schemes. It shows that for 2 antennas, our scheme gives nearly $2 \mathrm{~dB}$ gains over differential orthogonal space-time coding, whereas for 4 antennas, our scheme achieves $2 \mathrm{~dB}$ gains. Hence, our scheme has superior performance over other code schemes. Moreover, with the increase of the number of transmit antennas, the performance of differential codes with 4-antenna is superior to that with 2-antenna due to the increase of space diversity gains. Note that we do not provide 


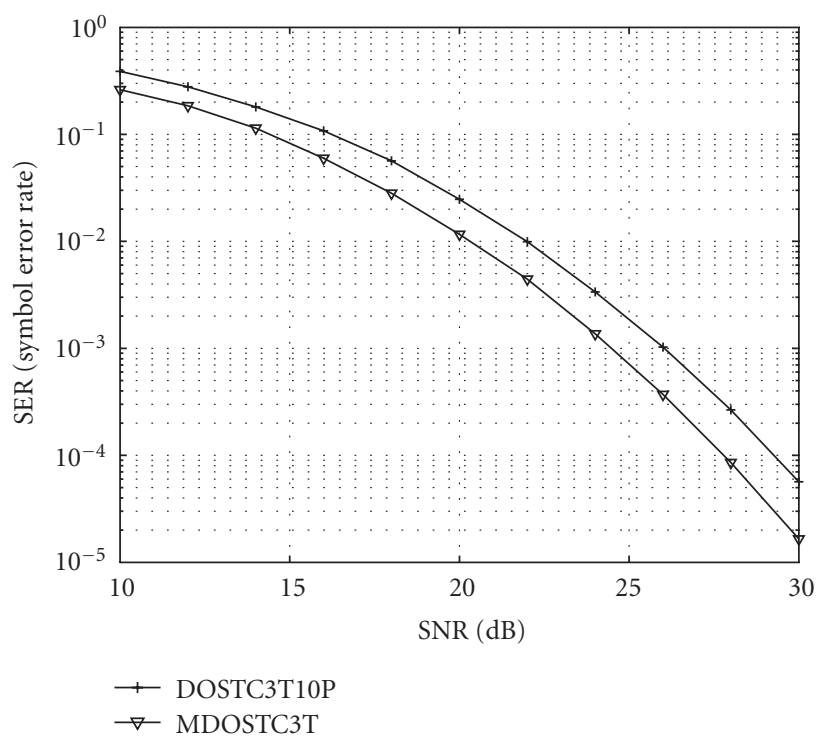

Figure 6: SER against SNR for DOSTC and MDOSTC with 3transmit antennas.

the performance comparison of differential unitary spacetime coding accordingly, since searching the optimal cyclic group code under the same spectrum efficiency is too difficult. In terms of the analysis method outlined in Section 4.3, the 3-transmit antennas performance comparison is provided in Figure 6. In this figure, the 16 star QAM method and 10 PSK are adopted. It is shown in Figure 6 that our scheme is still better than differential orthogonal space-time coding; it can obtain about $2 \mathrm{~dB}$ gains. Based on the above conclusions, our simulation results make an agreement with the previous theoretical analysis.

\section{CONCLUSIONS}

On the basis of differential unitary space-time coding and differential orthogonal space-time coding, by using the star QAM method, two kinds of multiple amplitudes differential space-time coding schemes are presented in this paper; one is multiple amplitudes differential unitary space-time coding; the other is multiple amplitudes differential orthogonal space-time coding. The two schemes can avoid the performance degradation of conventional DSTC scheme based on PSK modulation due to the decrease of minimum produce distance in high spectrum efficiency. The developed MDUSTC scheme can be applied to any number of antennas, and implement different data rates, and low-complexity differential modulation due to the application of cyclic group codes. It has higher coding gain than existing differential unitary space-time coding. For the developed MDOSTC scheme, it has higher coding gain than existing differential orthogonal space-time coding schemes. Moreover, it has simpler decoder and can obtain higher code rate in the case of three or four transmit antennas. The simulation results in fading channel also show that our schemes have lower BER than the corresponding differential unitary space-time codes and differential orthogonal space-time codes under the same SNR.

\section{ACKNOWLEDGMENTS}

This work is supported by the Chinese Jiangsu Planned Projects for Postdoctoral Research Funds and by China Postdoctoral Science Foundation under Grant no. 2005038242. The authors would like to thank the two anonymous reviewers for their useful comments and constructive suggestions throughout the course of reviewing this paper, and thank Prof. Richard Barton for his helpful suggestions.

\section{REFERENCES}

[1] N. Al-Dhahir, C. Fragouli, A. Stamoulis, W. Younis, and R. Calderbank, "Space-time processing for broadband wireless access," IEEE Communications Magazine, vol. 40, no. 9, pp. 136-142, 2002.

[2] V. Tarokh, N. Seshadri, and A. R. Calderbank, "Space-time codes for high data rate wireless communication: performance criterion and code construction," IEEE Transactions on Information Theory, vol. 44, no. 2, pp. 744-765, 1998.

[3] V. Tarokh, H. Jafarkhani, and A. R. Calderbank, "Space-time block codes from orthogonal designs," IEEE Transactions on Information Theory, vol. 45, no. 5, pp. 1456-1467, 1999.

[4] C. Gao and A. M. Haimovich, "BER analysis of MPSK spacetime block codes with differential detection," IEEE Communications Letters, vol. 7, no. 7, pp. 314-316, 2003.

[5] B. M. Hochwald and T. L. Marzetta, "Unitary space-time modulation for multiple-antenna communications in Rayleigh flat fading," IEEE Transactions on Information Theory, vol. 46, no. 2, pp. 543-564, 2000.

[6] B. M. Hochwald and W. Sweldens, "Differential unitary spacetime modulation," IEEE Transactions on Communications, vol. 48, no. 12, pp. 2041-2052, 2000.

[7] B. L. Hughes, "Differential space-time modulation," IEEE Transactions on Information Theory, vol. 46, no. 7, pp. 25672578, 2000.

[8] V. Tarokh and H. Jafarkhani, "A differential detection scheme for transmit diversity," IEEE Journal on Selected Areas in Communications, vol. 18, no. 7, pp. 1169-1174, 2000.

[9] H. Jafarkhani and V. Tarokh, "Multiple transmit antenna differential detection from generalized orthogonal designs," IEEE Transactions on Information Theory, vol. 47, no. 6, pp. 26262631, 2001.

[10] G. Ganesan and P. Stoica, "Space-time block codes: a maximum SNR approach," IEEE Transactions on Information Theory, vol. 47, no. 4, pp. 1650-1656, 2001.

[11] G. Ganesan and P. Stoica, "Differential modulation using space-time block codes," IEEE Signal Processing Letters, vol. 9, no. 2, pp. 57-60, 2002.

[12] J. G. Proakis, Digital Communications, McGraw-Hill, New York, 4th edition, 2001.

[13] M. Tao and R. S. Cheng, "Differential space-time block codes," in Proceedings of IEEE Global Telecommunications Conference (GLOBECOM '01), vol. 2, pp. 1098-1102, San Antonio, Tex, USA, November 2001.

[14] T. T. Tjhung, X. Dong, F. Adachi, and K. H. Tan, "On diversity reception of narrow-band 16 STAR-QAM in fast Rician 
fading," IEEE Transactions on Vehicular Technology, vol. 46, no. 4, pp. 923-932, 1997.

[15] Y. C. Chow, A. R. Nix, and J. P. McGeehan, "Analysis of 16APSK modulation in AWGN and Rayleigh fading channel," Electronics Letters, vol. 28, no. 17, pp. 1608-1610, 1992.

[16] A. Shokrollahi, B. Hassibi, B. M. Hochwald, and W. Sweldens, "Representation theory for high-rate multiple-antenna code design," IEEE Transactions on Information Theory, vol. 47, no. 6, pp. 2235-2367, 2001.

Xiangbin Yu received the M.S. degree in communication and information systems from Hohai University, Nanjing, China, in 2001; and his Ph.D. in communication and information systems in 2004 from the National Mobile Communications Research Laboratory at Southeast University, China. Now he is working as a Postdoctoral Researcher in Information and Communication Engineering Postdoctoral Research Sta-

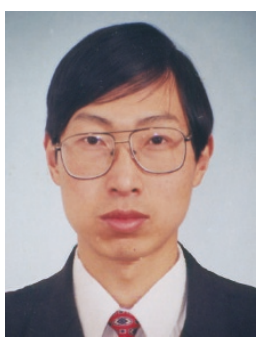
tion at Nanjing University of Aeronautics and Astronautics, Nanjing, China. His research interests include multicarrier digital communication, space-time coding, adaptive modulation, and digital signal processing in modern communications.

DaZhuan Xu was graduated from Nanjing Institute of Technology, Nanjing, China, in 1983. He received the M.S. degree and the $\mathrm{Ph} . \mathrm{D}$. in communication and information systems from Nanjing University of Aeronautics and Astronautics in 1986 and 2001, respectively. He is now a Full Professor in the College of Information Science and Technology, Nanjing University of Aeronautics and Astronautics, Nanjing, China.

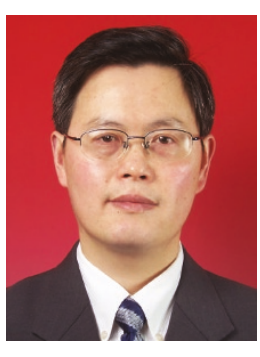
$\mathrm{He}$ is a Senior Member of China Institute of Electronics (CIE). His research interests include digital communications, software radio, coding theory, and medical signal processing.

Guangguo Bi was graduated from Nanjing Institute of Technology, Nanjing, China, in 1960. He is now a Professor at the National Mobile Communication Research Laboratory, the Department of Radio Engineering, Southeast University, Nanjing, China. He is a Fellow and a Member of the Board of Director of the China Institute of Communications, and a Senior Member of the IEEE. His research interests include digital com-

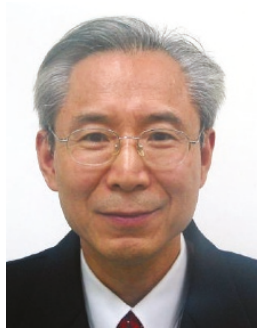
munications, personal communications network, spread spectrum communications, and intelligent information processing. He has published more than 200 papers in the above areas. 